\section{Estudo comparativo entre duas técnicas de tonsilectomia: bisturi harmônico (Ultracision) e dissecção tradicional com bisturi de lâmina fria}

Comparative study between two tonsillectomy techniques: Ultracision harmonic scalpel and traditional dissection with cold scalpel

Resumo / Summary

$\mathbf{T}$ onsilectomia é a cirurgia realizada com maior frequência na clínica otorrinolaringológica. As modificações e as evoluções da técnica ocorrem no sentido de simplificar o procedimento e minimizar as complicações. O bisturi harmônico (Ultracision) começou a ser utilizado na Otorrinolaringologia em 1999 para tonsilectomia com bons resultados. Objetivo: O objetivo deste estudo foi comparar o tempo cirúrgico, sangramento e hemostasia trans-operatórios, dor pós-operatória, aspecto cicatricial da loja tonsilar e intercorrências no trans e pós-operatório em pacientes submetidos a tonsilectomia pela técnica tradicional com lâmina fria e pela técnica utilizando lâmina cirúrgica em ganchos de coagulação Ultracision. Forma de estudo: Coorte transversal. Casuística e Método: Vinte e seis pacientes foram submetidos a tonsilectomia: 13 pela técnica tradicional com bisturi de lâmina fria e 13 pela técnica com Ultracision, avaliando os parâmetros previamente estabelecidos através de um protocolo padrão. Os pacientes foram submetidos a uma análise da intensidade da dor através da escala analógica visual horizontal. Resultados: O tempo cirúrgico foi estatisticamente menor na cirurgia realizada pela técnica com o bisturi harmônico comparada com a técnica tradicional. O percentual de pontos dados na loja tonsilar também mostrou-se mais baixo do que pela técnica tradicional. Não houve diferença significativa em relação ao padrão de dor e a evolução pós-operatória do aspecto cicatricial da loja. Conclusão: O bisturi harmônico mostrou ser um excelente recurso para a realização de cirurgias onde o tempo cirúrgico e o sangramento trans-operatório são de grande importância.
$\mathrm{T}$ onsillectomy is the most commom surgical procedure in Otolaringology. The modifications and the technique surgical evolutions aim to simplifly and reduce the complications. The UltacisionÒ harmonic scalpel begin to be used in 1999 in tonsillectomies with good outcomes. Aim: To compair the time of the procedure, bleeding and the need of trans operative hemostasis, post operative pain, healing aspect of the tonsilar fossa and complications in patients submitted to tonsillectomy with cold and ultrassonic scalpel. Study design: Transversal cohort. Material and Method: Twenty six patients underwent tonsillectomy: 13 using the traditional thecnique with cold instruments and 13 using the ultrassonic one. They were evaluated with a standart protocol. The post operative pain were graduated through the horizontal visual scale analogue. Results: Surgical time were shorter with the ultrassonic thecnique compared to the traditional cold instruments. The amount of stitch at tonsilar fossa was lesser than the traditional thecnique. There were no post operative statistical difference in pain and in the aspect of the tonsilar fossa. Conclusion: The ultrassonic scalpel is an excellent choice in surgeries where surgical time and transoperatory bleeding are important.

\footnotetext{
${ }^{1}$ Médico Otorrinolaringologista e Pós-Graduando da Disciplina de Otorrinolaringologia do Hospital das Clínicas da Faculdade de Medicina da USP - Ribeirão-Preto - SP. ${ }^{2}$ Médicos Residentes do Núcleo de Otorrinolaringologia e Cirurgia de Cabeça e Pescoco de São Paulo.

${ }^{3}$ Diretor do Núcleo de Otorrinolaringologia e Cirurgia de Cabeça e Pescoço de São Paulo e Chefe do Serviço de Otorrinolaringologia do Hospital e Maternidade São Camilo - São Paulo.

Trabalho realizado na Clínica Dr. Fernando Arruda Ramos em conjunto com o Núcleo de Otorrinolaringologia e Cirurgia de Cabeça e Pescoço de São Paulo. Trabalho apresentado no $36^{\circ}$ Congresso Brasileiro de Otorrinolaringologia, Florianópolis - Santa Catarina - SC.

Endereço para correspondência: Fernando Arruda Ramos - Rua Lauro Muller, $5472^{\circ}$ andar, Lages Santa Catarina SC 88501130

Fone/Fax (0xx49) 222-9165 - E-mail: arrudaramos@matrix.com.br

Artigo recebido em 24 de março de 2003. Artigo aceito em 29 de abril de 2004.
} 


\section{INTRODUÇÃO}

A cirurgia de extirpação das tonsilas palatinas é uma das mais antigas do mundo, com relatos de sua ocorrência já no ano 3 D.C. realizada por Cornelius Caesus. ${ }^{1}$

Várias técnicas de tonsilectomia já foram descritas, dentre elas a disseção a frio, a dissecção com guilhotina, dissecção bipolar, dissecção com laser e a técnica da tesoura bipolar. $^{2}$

A tonsilectomia permanece sendo a cirurgia mais comum dentro da otorrinolaringologia, e todos estes avanços técnicos vem objetivando uma menor morbidade para o procedimento.

Atualmente, as indicações da tonsilectomia estão bem definidas e são divididas em absolutas e relativas. As indicações absolutas são: hipertrofia das tonsilas palatinas com obstrução respiratória superior, Síndrome da ApnéiaHipopnéia Obstrutiva do Sono (SAHOS), suspeita de malignidade, tonsilite hemorrágica, déficit de crescimento, anormalidades de linguagem, dificuldade de alimentação, má oclusão dentária e anormalidades do crescimento orofacial. As relativas são: tonsilites recorrentes, tonsilites associadas com doenças sistêmicas (portador do Streptococcus Betahemolítico do grupo A), abscesso peritonsilar, halitose por tonsilite caseosa..$^{3-6}$

O aparelho bisturi ultracison ou bisturi harmônico está indicado para realizar incisões em tecidos moles, quando se pretende controlar hemorragias e minimizar as lesões térmicas, complementando ou substituindo o eletrocautério, laser ou até mesmo o bisturi de lâmina fria.

Procedimentos na área de Ginecologia, Urologia, Cirurgia Torácica e Laparoscópica já se utilizam do bisturi harmônico há alguns anos².

O seu uso foi introduzido na Otorrinolaringologia em 1999 para tonsilectomia microscópica com bons resultados. Este bisturi apresenta energia mecânica vibratória com freqüência em torno de $55,5 \mathrm{khz}$ e atua promovendo corte e coagulação a depender do nível de potência ( 1 a 5), tipo de técnica, fração do tecido e pressão da lâmina.

O gancho dissector é adotado como padrão para realização das tonsilectomias. Em geral, instrumentos de superfície mais romba proporcionam uma melhor coagulação e cortam mais lentamente. A quantidade de energia fornecida aos tecidos e os seus efeitos sobre o mesmo dependem de diferentes fatores como: nível de potência selecionado, características da lâmina, tensão tecidual, tipo de tecido, patologia e a técnica cirúrgica. ${ }^{7}$ Quanto maior a potência do aparelho, maior a vibração e conseqüentemente, maior a área de corte ${ }^{2}$.

O uso do bisturi Ultracision constitui uma inovação no tratamento cirúrgico das tonsilas palatinas. Apresentamos este estudo para demonstrarmos a sua utilização, vantagens e desvantagens, comparando-o com a técnica tradicional de dissecção com instrumental a frio, tendo como principais parâmetros: tempo cirúrgico, sangramento e hemostasia no trans-operatório, padrão de dor e aspecto da loja tonsilar no pós-operatório e intercorrências no trans e pós-operatório.

\section{CASUÍSTICA E MÉTODO}

Foram operados 26 pacientes, divididos randomicamente em dois grupos: 13 submetidos a técnica com uso de lâmina em gancho de coagulação, Ultracision (Grupo 1) e 13 submetidos a técnica de dissecção com bisturi de lâmina fria e aspirador-descolador (Grupo 2). As idades mínima e máxima foram respectivamente de 3 e 20 anos. A idade dos pacientes do grupo 1 variou de 4 a 16 anos (idade média de 6,53 anos) e do grupo 2 variou de 3 a 20 anos (idade média de 7,5 anos). Seis dos 13 pacientes operados por ambas as técnicas eram do sexo masculino, e 7 do sexo feminino. (Tabela 1). Hipertrofia de tonsilas com obstrução respiratória superior e SAHOS foram os critérios de inclusão. Os critérios de exclusão foram: tonsilites recorrentes, abscesso peritonsilar prévio e tonsilite crônica caseosa. Todos os pacientes avaliados foram incluídos no estudo, não havendo nenhuma abstenção.

As cirurgias foram realizadas nos seguintes hospitais: Hospital Geral e Maternidade Tereza Ramos e Hospital Nossa Senhora dos Prazeres, ambos na cidade de Lages, Santa Catarina. O estudo foi aprovado pelo comitê de ética médica de ambos os hospitais. O aparelho bisturi Ultracision ou bisturi harmônico, produzido pela Johnson \& Johnson, foi nos concedido pela Ethicon Endo-Surgery. Este bisturi apresenta energia mecânica vibratória com freqüência em torno de 55,5 Khz e atua promovendo corte e coagulação a depender do nível de potência ( 1 a 5), tipo de técnica, fração do tecido e pressão da lâmina. O instrumental esterelizado destina-se a um único paciente e é composto por uma lâmina de titânio com uma bainha não removível. Os instrumentais de $5 \mathrm{~mm}$ estão disponíveis com três tipos de lâminas: um gancho dissector, um gancho dissector de ponta afiada e uma lâmina curva. Todas as lâminas possuem um comprimento de $14 \mathrm{~cm}^{2,7}$. Foi adotado como padrão para realização das tonsilectomias o gancho dissector.

Todas as cirurgias foram realizadas pelo mesmo cirurgião, sendo que houve um período de treinamento específico (curva de aprendizagem) para desenvolver a técnica com o Ultracision. O cirurgião realizou, antes de iniciar o

Tabela 1. Dados demográficos

\begin{tabular}{lcc}
\hline & masculino & feminino \\
\hline Ultracision & 6 & 7 \\
Lâmina fria & 6 & 7 \\
\hline
\end{tabular}

Nota: valores sem diferenças estatisticamente significantes para o teste "t" de Student com nível de significância de 5\% 
trabalho, uma série de 10 cirurgias com esta técnica no Hospital e Maternidade São Camilo - Pompéia, na cidade de São Paulo, em conjunto com a equipe do Núcleo de Otorrinolaringologia e Cirurgia de Cabeça e Pescoço de São Paulo e os técnicos responsáveis pela manutenção do aparelho, treinados pelo fabricante.

Foram analisados os seguintes parâmetros cirúrgicos através de um protocolo previamente estabelecido: tempo cirúrgico, sangramento trans e pós-operatório, hemostasia, padrão de dor e aspecto da loja no pós-operatório, medicação e intercorrências trans e pós operatórias. (Figura 1)

Todos os pacientes foram entubados via orotraqueal, sob anestesia geral inalatória. O tempo de cada cirurgia era contado a partir do momento da incisão, independente da técnica, até o momento em que a hemostasia era satisfatória, através de sutura ou apenas pela compressão da loja com gaze molhada em soro fisiológico. Após a dissecção da amígdala esquerda, fazia-se a adenoidectomia com cureta de Beckmann, quando havia indicação para tal. Trocava-se o tubo orotraqueal de lado. Após a exposição da tonsila palatina direita, iniciava-se a incisão contando novamente o tempo cirúrgico desse lado. A hemostasia foi realizada, quando necessário, por compressão com gaze por um período de aproximadamente 2 minutos e com fio tipo Categute 2.0 simples com agulha de $2 \mathrm{~cm}$.

Para comparação das técnicas, foi aplicado um protocolo previamente estabelecido em todas as crianças operadas por ambas as técnicas. Foi avaliado através do protocolo o tempo cirúrgico e a hemostasia de cada lado isoladamente, considerando a necessidade ou não de sutura em cada loja. Na análise estatística foi considerado o tempo total, ou seja, a soma do tempo de ambos lados. Se houvesse algum tipo de intercorrência durante as cirurgias, essa seria relatada separadamente.

A primeira avaliação no pós-operatório foi realizada na sala de recuperação pós-anestésica, onde foram analisados a presença ou não de sangramento e a existência ou não de dor. A segunda avaliação pós-operatória foi realizada em torno de 10 horas após a cirurgia, no momento da alta hospitalar. Nesse segundo momento, avaliou-se a intensidade da dor através da Escala Analógica Visual (EAV) e o aspecto das lojas tonsilares. A EAV com orientação horizontal consiste de desenhos lineares com 7 faces, 1 face neutra e 6 faces com expressão de sensação crescente de dor. Estas faces estão ordenadas de forma crescente de 1 a 7 , da esquerda para direita. Sem dor (escore 1) na extremidade esquerda e dor de intensidade máxima (escore 7) na extrema direita. O escore subjetivo é igual à escala numérica, numerado de 1 a 7 . (Figura 2)

A alta hospitalar era dada com orientação para as mães das crianças operadas em relação à administração das escalas, a dieta, o uso de medicamentos padronizados e repouso relativo.

Os pacientes foram orientados a retornar no quarto ou quinto dia pós-operatório para a primeira avaliação, e o segundo retorno por volta do décimo dia após a cirurgia. O acompanhamento pós-operatório de todos os pacientes foi realizado pelo mesmo cirurgião. Em cada retorno, fazia-se a análise das EAVs devidamente preenchidas e exame otorrinolaringológico, sendo também questionados em relação à presença de alguma intercorrência.

\section{Escala Analógica Visual (EAV)}

Testou-se primeiramente, no pré-operatório, a compreensão da EAV da dor. Questionou-se ao paciente e/ou responsável a face que demonstrava mais e menos dor.

Em seguida, a EAV era apresentada ao paciente no momento da alta hospitalar, o qual era solicitado a mostrar qual o ponto da escala da dor facial que melhor representava a sua sensação dolorosa da orofaringe, verificando-se a compreensão da mesma pelo paciente e pelo acompanhante e avaliando-se a intensidade da dor nesse primeiro instante (Figuras 2 e 3).

O paciente e/ou responsável foram orientados para o preenchimento dessa escala diariamente, logo após acordar, sem ainda ser administrado o analgésico durante um período de dez dias.

Os pacientes fizeram acompanhamento pós-operatório no quarto ou quinto dia após a cirurgia, onde levavam as EAVs correspondentes a esse período e eram orientadas a retornarem pelo menos mais uma vez para nova avaliação.

\section{Evolução pós-operatória}

O aspecto da loja tonsilar foi avaliado de acordo com o protocolo, através da análise da loja direita e da loja esquerda isoladamente nos seguintes aspectos: presença ou não de edema, fibrina, coágulo, loja tonsilar seca ou com sangramento focal ou difuso. A primeira avaliação da loja tonsilar em relação aos aspectos expostos acima foi durante a alta hospitar.

Para cada retorno foi aplicado o protocolo através do qual verificava-se o escore da EAV e o exame físico otorrinolaringológico, avaliando-se o aspecto da loja. Foi avaliado conjuntamente se houve alguma intercorrência no período entre as consultas como quadros de náuseas e/ou vômitos, otalgia reflexa ou cefaléia e questionou-se quanto ao uso do analgésico padronizado e sua eficácia.

Para analgesia pós-peratória, padronizou-se o Paracetamol na dosagem correspondente ao peso: uma gota por Kg de peso (solução com $100 \mathrm{mg} / \mathrm{ml}$ ) se menos de 40 $\mathrm{kg}$, ou 1 comprimido de $750 \mathrm{mg}$ se mais de $40 \mathrm{Kg}$, até de 4 em 4 horas. Em caso de febre (temperatura maior ou igual a $37,8^{\circ} \mathrm{C}$ ), orientou-se o uso da mesma medicação com dosagem e posologia semelhantes.

Todos os pacientes foram orientados em relação aos cuidados pós-operatórios (evitar esforço físico, exposição ao calor e banhos quentes) necessários para que não houvesse fatores externos que comprometessem a avaliação da evolução pós-operatória. Foi introduzida uma dieta líquida, cremosa, morna e/ou fria até a primeira avaliação. 
PROTOCOLO DO ESTUDO COMPARATIVO ENTRE DUAS TÉCNICAS DE TONSILECTOMIA: BISTURI HARMÔNICO (ULTRACISON) E DISSECÇÃO TRADICIONAL COM BISTURI DE LÂMINA FRIA

Nome:

Idade:

Hospital:

Cirurgia:

Técnica utilizada

Tempo cirúrgico

Tempo hemostasia

Intercorrências

Ultracisionpotência

Pós-op imediato (RPA)

. Sangramento

Ultracision

esq

esq

esq

intrumental a frio

dir

dir

dir

. Dor

Pós-op imediato (alta hospitalar)

. Dor

. EAV:

. Oroscopia

Aspecto da loja

Edema

Fibrina

Sangramento

Coágulo

Seca

Intercorrências

Medicação

Analgésico

Freqüência

Eficácia

Outros

Avaliação $4^{\circ} / 5^{\circ}$ dia pós-op

Dor

EAV:

Aspecto da loja

Edema

Fibrina

Sangramento

Coágulo

Seca

Intercorrências

Medicação

Analgésico

Freqüência

Eficácia

Outros

sim

não

dir

sim

dir

esq

não

esq

Hora

$\operatorname{sim}$

não

dir

esq

Avaliação $10^{\circ} / 11^{\circ}$ dia pós-op

Dor

dir

sim não

sim não

sim não

sim não

sim não

esq

sim não

sim não

sim não

sim não

sim não

EAV:

Aspecto da loja

Edema

Fibrina

Sangramento

Coágulo

Seca

Intercorrências

Medicação

Analgésico

Freqüência

Eficácia

Outros

$\operatorname{sim}$

dir

sim

$\operatorname{sim}$

$\operatorname{sim}$

sim

$\operatorname{sim}$

$\operatorname{sim}$

dir esq

sim não

sim não

sim não

sim não

sim não não

$\begin{array}{ll}\text { sim } & \text { não } \\ \text { sim } & \text { não } \\ \text { sim } & \text { não } \\ \text { sim } & \text { não } \\ \text { sim } & \text { não }\end{array}$

não

sim não

sim não

sim não

sim não

sim não

Figura 1. Protocolo de avaliação pós-operatória 


\section{RESULTADOS}

Dos 26 pacientes incluídos neste estudo, apenas um operado com Ultracision foi submetido à tonsilectomia isolada. Todos os demais foram submetidos a adenotonsilectomia.

A potência 2 foi utilizada em $77 \%$ dos pacientes operados com o bisturi Harmônico. Nos demais, utilizou-se a potência 3 .

O tempo médio de cirurgia para a técnica com Ultracision (grupo 1) foi de 14,92 minutos, com desvio padrão de 3,38, e o tempo médio da cirurgia com instrumentos a frio (grupo 2) foi de 25,46 minutos, com um desvio padrão de 3,82. Essa diferença entre os tempos cirúrgicos foi estatisticamente significativa pela análise do teste $\mathrm{T}$ de Student, com $\mathrm{P}<0,05$. (Tabela 2)

Houve sangramento durante a cirurgia em apenas 15,4\% dos pacientes do grupo 1 , necessitando fazer hemostasia com fio Categute simples 2.0. Todos os pacientes do grupo 2 necessitaram de hemostasia com o mesmo fio.

Na avaliação pós-operatória dentro da recuperação pós-anestésica (RPA), não houve sangramento em nenhum dos grupos. A dor esteve presente em 30,8\% dos pacientes do grupo 1 e em $7,7 \%$ dos pacientes do grupo 2 .

Todos os pacientes tiveram alta num período de até 12 horas após a cirurgia. A avaliação durante a alta hospitalar mostrou os seguintes dados, em relação ao aspecto da loja:

edema esteve presente em 92,3\% dos pacientes do grupo 1 e em 61,5\% do grupo 2; 100\% dos pacientes do grupo 1 e $23 \%$ dos pacientes do grupo 2 tinham fibrina nas lojas. Nenhum dos pacientes de ambos grupos tiveram san-

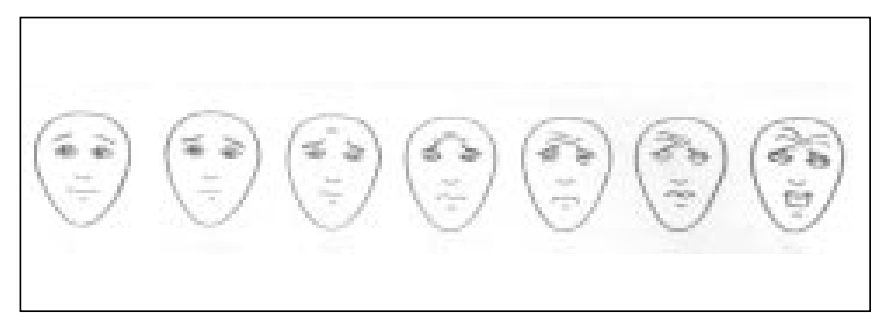

Figura 2. Escala analógica visual horizontal

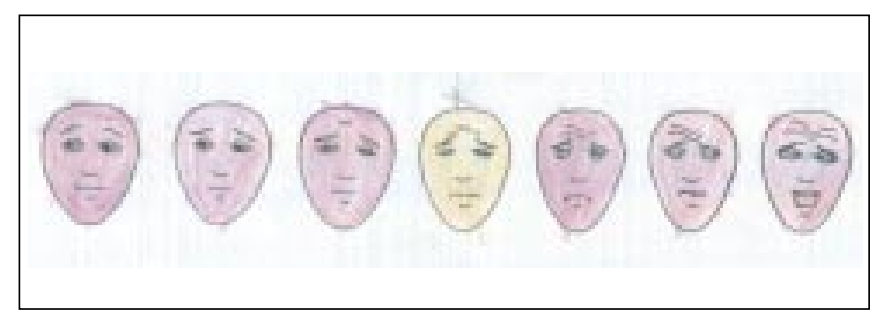

Figura 3. Escala analógica visual preenchida por uma criança no $4^{\circ}$ dia pós-operatório submetida a tonsilectomia com bisturi harmônico (Ultracison) gramento neste período. Um paciente (7.7\%) do grupo $1 \mathrm{e}$ $5(38,5 \%)$ do grupo 2 tinham coágulo nas lojas tonsilares. Somente um paciente do grupo 1 teve alguma intercorrência, no caso vômitos. (Tabela 3)

Os resultados da avaliação no $4^{\circ}$ ou $5^{\circ}$ dia pós-operatório são os seguintes: $69.24 \%$ dos pacientes do grupo $1 \mathrm{e}$ $30,8 \%$ dos pacientes do grupo 2 apresentavam edema. Todos os pacientes do grupo 1 e 46,1\% dos pacientes do grupo 2 tinham fibrina nesse período. Somente um paciente do grupo $1(7,7 \%)$ referiu sangramento e nenhum no grupo 2 . Um paciente de cada grupo apresentava coágulos nas lojas. Cinco pacientes do grupo $1(38,5 \%)$ e 2 pacientes do grupo 2 $(15,4 \%)$ referiram ter tido algum tipo de intercorrência (otalgia reflexa, náuseas e vômitos). (Tabela 4)

Quanto às avaliações no $9^{\circ}, 10^{\circ}$ ou $11^{\circ}$ dias de pósoperatório: $38 \%$ dos pacientes apresentavam edema leve no grupo 1 , e $7,7 \%$ apresentavam edema no grupo 2; 69\% dos pacientes do grupo 1 e $46 \%$ dos pacientes do grupo 2 tinham fibrina na loja. Nenhum paciente referiu sangramento. Um paciente do grupo 1 apresentava coágulos nas lojas. Três pacientes (23\%) do grupo 1 referiram ter tido alguma intercorrência (otalgia). Não houve relato de intercorrências no grupo 2 .

\section{DISCUSSÃO}

Discussões sobre vantagens e desvantagens de diferentes técnicas de tonsilectomia são frequentes. ${ }^{11,12}$

Numa casuística de 26 pacientes, que foram submetidos a cirurgia de tonsilectomia pela técnica com o bisturi harmônico, D'Ávila relata os seguintes resultados: a duração

Tabela 2. Tempo cirúrgico médio e desvio padrão para ambas técnicas.

\begin{tabular}{lc}
\hline & Média (desvio padrão) \\
\hline Ultracision & $14,92(3,38)$ \\
Lâmina fria & $25,46(3,82)$ \\
\hline
\end{tabular}

Nota: valores com diferenças estatisticamente significantes para o teste " $\mathrm{t}$ " de Student com nível de significância de $5 \%$

Tabela 3. Média e desvio padrão da dor na alta hospitalar.

\begin{tabular}{lc}
\hline & Média (desvio padrão) \\
\hline Ultracision & $3,04(2,35)$ \\
Lâmina fria & $4,55(2,18)$ \\
\hline Nota: $p=0,08$ &
\end{tabular}

Tabela 4. Média e desvio padrão da dor no $4^{\circ}$ e $5^{\circ}$ dia.

\begin{tabular}{lc}
\hline & Média (desvio padrão) \\
\hline Ultracision & $2,9(2,25)$ \\
Lâmina fria & $2,66(1,5)$ \\
\hline Nota: valores sem diferenças estatisticamente significantes para \\
o teste "t" de Student com nível de significância de $5 \%$
\end{tabular}


do ato cirúrgico para cada tonsila foi de 14 minutos. Houve excelente controle do sangramento trans-operatório em quase todos os vasos da região tonsilar. No pós-operatório imediato e tardio não ocorreram complicações. Não houve hemorragias. A dor pós-operatória foi um sintoma pouco referido por esse grupo de pacientes. ${ }^{13}$

Sood et al., em seu trabalho com 158 tonsilectomias das palatinas em 59 pacientes utilizando o bisturi harmônico encontrou uma diferença estatística em relação ao tempo do procedimento cirúrgico e ao volume do sangramento no trans-operatório em comparação com a dissecção utilizando bisturi de lâmina fria ${ }^{2}$.

Nosso estudo também mostra uma diferença significativa entre as técnicas, em relação ao tempo cirúrgico. O tempo médio de cirurgia pela técnica com o bisturi harmônico foi de 14,92 minutos e o tempo médio pela técnica tradicional de dissecção com lâmina fria foi de 25,46 minutos.

Não houve diferença significativa entre a idade e o sexo dos pacientes operados de cada grupo em relação a dor, fato este que poderia ser um fator de confusão, pois estudos mostram diferenças entre os padrões de dor em diferentes idades ${ }^{12}$.

A avaliação da dor pós-operatória através do uso da escala analógica visual (EAV), constitui o método mais amplamente utilizado para avaliação da intensidade e da sensação subjetiva da dor $^{8}$. A EAV pode ser vertical ou horizontal, de acordo com a sua linha de orientação. Estudos comparando a orientação vertical e horizontal das EAVs mostram resultados controversos 9 . Em 1998, Breivik et al. demonstraram sensibilidade semelhante entre EAV vertical e horizontal na avaliação da dor após cirurgia da cavidade oral.

A avaliação da dor em crianças pequenas é bastante discutida em relação a sua confiabilidade devido a fatores como expressão e linguagem. As EAVs podem mostrar a avaliação da dor nos pacientes com idade abaixo dos sete anos. Crianças abaixo desta faixa etária compreendem completamente o mecanismo das mesmas. A escala da dor facial aplicada neste estudo pode ser usada com segurança em crianças de baixa idade em relação à compreensão e concepção. $^{10}$

Pacientes com doença de Alzheimer possuem dificuldades de expressão e de linguagem semelhantes a crianças de baixa idade. Num estudo realizado com pacientes portadores da doença de Alzheimer, com grau leve e moderado do acometimento desta patologia, observou-se que este modelo de avaliação constitui uma ferramenta segura para avaliação da intensidade e afetividade da dor. Nos pacientes que compreendem completamente o propósito da escala da dor facial, faz-se bem a avaliação da dor. O mesmo pode ser dito das crianças nas quais se aplica a escala. ${ }^{10}$

Em relação ao estudo de Sood et al., o próprio autor afirma que ficou impressionado com a ausência de dor no pós-operatório imediato, pois a maioria dos pacientes já estava se alimentando nas primeiras horas após o procedimento. Entretanto, a dor nos dias subseqüentes não foi estatisticamente diferente em relação ao bisturi a frio ${ }^{2}$. A ausência de dor intensa no pós-operatório imediato assim como um menor tempo de sala no Centro Cirúrgico justificariam a utilização do aparelho, já que seu custo operacional é bem mais elevado que a dissecção com lâmina fria, uma vez que miniminiza os custos hospitalares com uma alta hospitalar mais precoce ${ }^{2}$.

No presente estudo, a dor no pós-operatório imediato (recuperação pós-anestésica) esteve presente no grupo 1 (30,8\% dos casos) e foi maior do que no grupo $2(7,7 \%)$.

Utilizou-se a potência 2 na maioria dos pacientes submetidos a tonsilectomia com bisturi harmônico (77\%), pois quanto menor a intensidade da potência, maior é o potencial de coagulação e menor o corte. A cirurgia realizada com essa potência mostrou ser mais eficiente para a dissecção da tonsila palatina, pois transmite maior segurança em relação a hemostasia tanto no trans quanto no pós-operatório. Dos pacientes que necessitaram de sutura para hemostasia durante a cirurgia, metade deles estavam sendo operados na potência 3 .

O menor tempo cirúrgico apresentado pela técnica com bisturi harmônico pode estar relacionado ao fato dessa técnica realizar ao mesmo tempo corte e coagulação, sendo mínimo o sangramento comparado com a técnica tradicional. Com a diminuição do sangramento, apenas 15\% dos pacientes operados por essa técnica necessitaram de sutura nas lojas para hemostasia, ganhando-se um tempo considerável para a finalização da cirurgia.

\section{CONCLUSÃO}

O bisturi harmônico apresenta vantagens em relação à técnica tradicional de dissecção com instrumental a frio, tais como: corte e coagulação simultâneos, otimizando o tempo cirúrgico. Outros fatores positivos incluem:

- A não utilização de corrente elétrica (sem riscos de queimaduras);

- A mínima carbonização tecidual;

- Fácil instalação e manipulação do aparelho.

Observamos que não houve diferença significativa em relação ao padrão de dor e a evolução pós-operatória (aspecto cicatricial da loja tonsilar e intercorrências no pósoperatório). O menor tempo cirúrgico e como conseqüência um menor tempo de sala cirúrgica, a menor necessidade de sutura da loja tonsilar para hemostasia e a boa evolução pós-operatória demonstram tratar-se de uma técnica segura e eficaz que, quando disponível, minimiza os custos hospitalares, podendo assim substituir a técnica tradicional de dissecção com bisturi de lâmina fria. 


\section{AGRADECIMENTOS}

Nós agradecemos ao Dr. Fábio Eduardo Festugato pela realização das análises estatísticas e a Mariel Wahrhastig, Gabriela Dias e José Eduardo Passos da Johnson \& Johnson, que nos concederam o aparelho Ultracision e seus instrumentais.

\section{REFERÊNCIAS BIBLIOGRÁFICAS}

1. Ramos CM, Gonçalves EMR, Mendonça RR, Gualandro DM. Tonsilectomia: Técnica de dissecção X Técnica de Sluder. Rev Bras Otolaringol 2001; 67(2):229-32.

2. Sood S, Corbridge R, Powles J, Bates G, Newbegin CJR. Effectiveness of the ultrasonic harmonic scalpel for tonsillectomy. ENT - Ear, nose \& Throut Journal 2001August: 514-8.

3. Kavanagh TK, Beckford SN. Adenotonlillectomy in children: Indications and Contraindications. Southern Medical Journal 1988; 81(4): 507-11.

4. Ahlqvist-Rastad J, Hultcrantz E, Svanholm H. Children with Tonsilar Obstruction: Indications for and Efficacy of Tonsillectomy. Acta Paediatr Scand 1988; 77: 831-5.
5. Suen SJ, Arnold EJ, Brooks JL. Adenotonsillectomy for treatment of Obstructive Sleep Apnea in Children. Arch Otolaryngol Head and Neck Surg 1995; 121: 525-30.

6. Almeida ER, Campos VAR - In Campos CAH, Costa HOO. Tratado de Otorrinolaringologia. $1^{\text {a }}$ edição. São Paulo: Ed Rocca; vol 3: 248-52, 2002.

7. Fenton RS, Long J. Ultrasonic Tonsillectomy. The journal of Otolaryngol 2000: 29(6)

8. Breivik EK, Skoglund LA. Comparison of Present Pain Intensity Assessments on Horizontally and Vertically Oriented Visual Analogue Scales. Meth Find Exp Clin Pharmacol 1998; 20(8): 719-24.

9. Sriwantanakul K, Kelvie W, Lasagna L. The quantification of pain An analysis of words used to describe pain and analgesia in clinical trials. Clin Pharmacol Ther 1982; 32: 143-8.

10. Scherder EJA, Bouma A. Visual Analogue Scales for Pain Assessment in Alzheimer's Disease. Gerontology 2000; 46: 47-53.

11. Monteiro E. Amigdalectomia: dissecção x sluder. Rey BrasOtolaringol 2001; 67(3): 319-24.

12. Sant'Anna GD. Dor pós-tonsilectomia: comparação entre pacientes com diferentes idades. Revista Brasil Orl 2000; 66(2): 123-7.

13. D'Avila JS. Microcirurgia de tonsilas com bisturi Ultracision. Arq Fund Otorrinolaringol 2001; 5(4) 